\title{
Supplements: Development of the drop Freezing Ice Nuclei Counter (FINC), intercomparison of droplet freezing techniques, and use of soluble lignin as an atmospheric ice nucleation standard
}

\author{
Anna J. Miller ${ }^{1, *}$, Killian P. Brennan ${ }^{2, *}$, Claudia Mignani ${ }^{3}$, Jörg Wieder ${ }^{2}$, Robert O. David ${ }^{4}$, and \\ Nadine Borduas-Dedekind ${ }^{1,2}$ \\ ${ }^{1}$ Institute for Biogeochemistry and Pollutant Dynamics, ETH Zurich, Zurich, 8092 Switzerland \\ ${ }^{2}$ Institute for Atmosphere and Climate Science, ETH Zurich, Zurich, 8092 Switzerland \\ ${ }^{3}$ Institute of Environmental Geosciences, University of Basel, Basel, 4056 Switzerland \\ ${ }^{4}$ Department of Geosciences, University of Oslo, Oslo, 0315 Norway \\ ${ }^{*}$ These authors contributed equally to this work.
}

Correspondence: Nadine Borduas-Dedekind (nadine.borduas@usys.ethz.ch, @ nadineborduas)

Copyright statement. TEXT

Contents

S1 Overview of existing drop-freezing instruments

S2 Freezing detection

5 S3 Pre-treatment of Piko PCR trays

S4 Temperature calibration experiments

S5 Limit of detection

S6 Volume dependence on non-homogeneous freezing

S7 Effect of water background contamination

S8 Water condensation calculations

S9 Well surface area calculations

S10Effect of bubbles in the well 


\section{S1 Overview of existing drop-freezing instruments}

We complied an overview of bench-top droplet freezing techniques (DFTs), accessible in the document entitled 'dropfreezinginstruments.csv' in the ETH Research Collection at https://doi.org/10.3929/ethz-b-000438875. We have limited the list, to be relevant and concise, to instruments which 1) were developed or used for atmospheric ice nucleation measurements, 2) measure more than one droplet per measurement, and 3) were published in the year 2000 or later. The file includes for each of the 41

20 instruments: a) its full name, b) its acronym, c) the cooling method used, d) the generation of sample volume, e) the freezing detection method, f) its temperature range, $g$ ) the droplet size, h) the number of droplets per experiment, i) the year it was first published, j) the location of the device, $k$ ) the reference associated with its first publication, and l) a link to the publication. 


\section{S2 Freezing detection}

The freezing detection on FINC works with images of the Piko PCR trays, taken every $2{ }^{\circ} \mathrm{C}$ by a camera. Well location detection

is implemented with the Circular Hough transform algorithm similarly described in David et al. (2019). The automatic well alignment is shown in Figure S1. Since the automatic well detection was not always reliable, we also developed a filtered algorithm output to identify the well positions, as well as the option for a manual well alignment grid by the user selecting two opposing corner wells. For data visualization, a color map of the freezing temperature is also generated to visually inspect any well location bias $\mathrm{S} 2$.

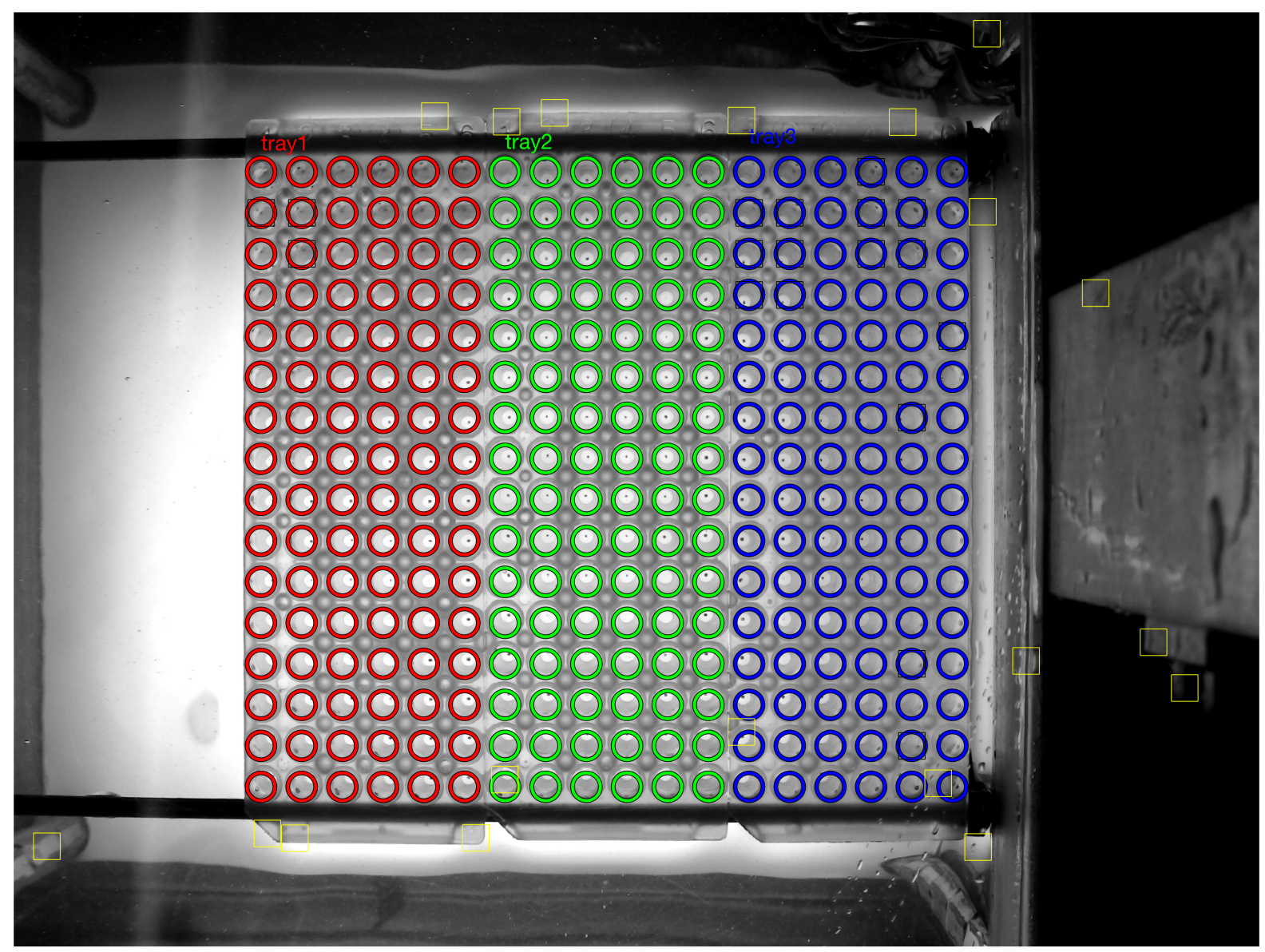

Figure S1. Automatic well alignment. The black and white photograph is taken from the camera on FINC of the three PCR trays. The colored circles overlaying the image are the automatic detected wells of the different trays. Yellow squares show misdirected wells, which is purely prognostic and does not affect freezing analysis. 


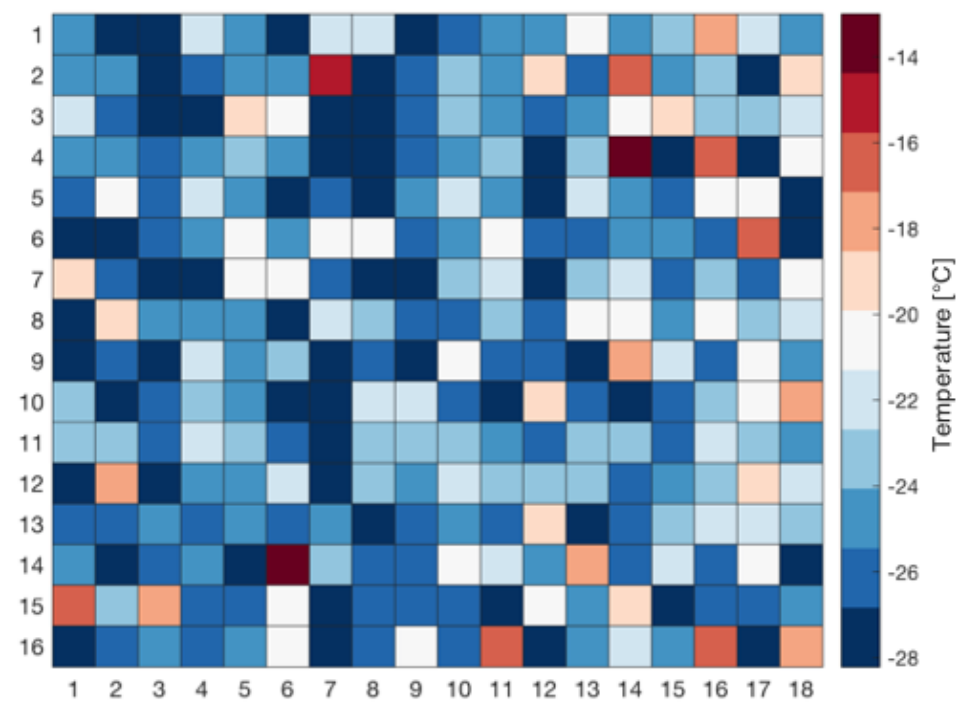

Figure S2. Map of freezing temperatures for each well. Each square represents the location of the 288 wells. The color scale represents the temperature at which each well froze (red colors for warmer freezing temperatures, blue colors for colder freezing temperatures). 
Freezing experiments with background water in Piko PCR trays used directly from the package showed irreproducible results (black markers in Figure S3). After pre-treating the trays by heating them in an oven at $120^{\circ} \mathrm{C}$ for at least one hour, background water experiments were more reproducible (red markers in Figure S3).

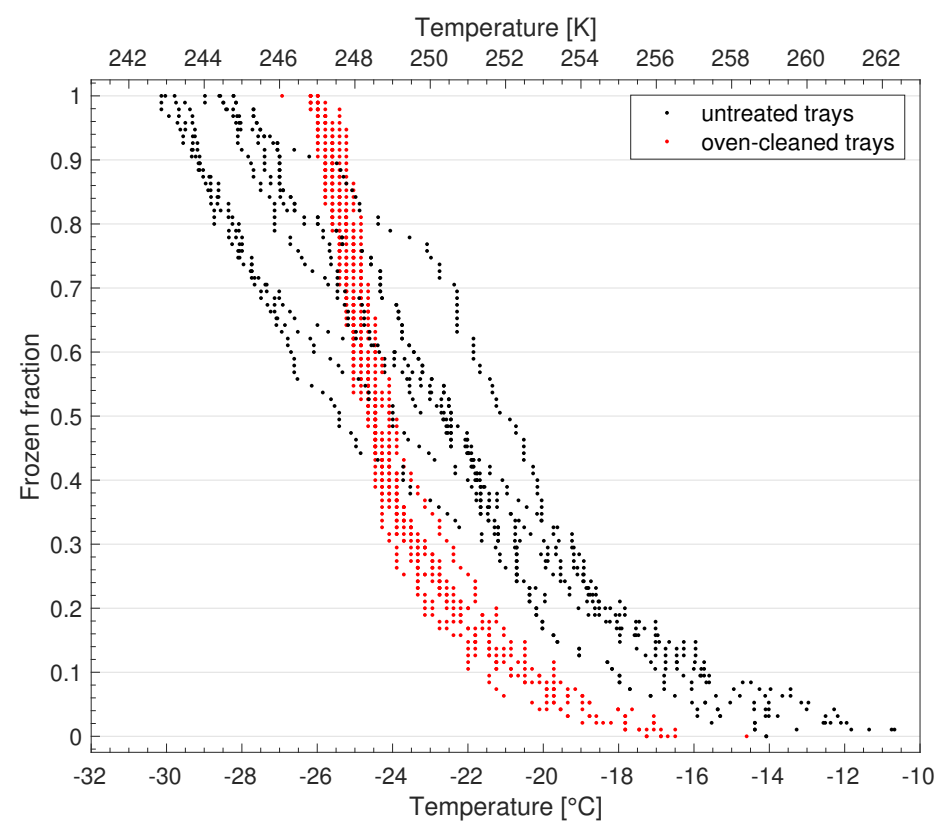

Figure S3. Frozen fraction curves of untreated Piko PCR trays and oven-cleaned Piko PCR trays. Each curve represents one 96-well tray. Untreated trays were used directly from the package (black dots), whereas the oven-cleaned trays were placed in the oven at $120{ }^{\circ} \mathrm{C}$ for at least one hour prior to pipetting in sample (red dots). These experiments used $20 \mu \mathrm{L}$ of molecular biology free reagent water. All temperature measurements have an uncertainty of $\pm 0.5^{\circ} \mathrm{C}$. 


\section{S4 Temperature calibration experiments}

35 A number of tests were conducted to see how the tray number, tray position, and the bath pump speed would affect the temperature inside the wells of the tray in FINC. We used a multi-channel thermocouple data logger (HH-4208SD, Thermosense). Each K-type thermocouple was placed inside a well filled with ethanol, and the thermocouples were evenly spaced across the trays in the bath. For each test, the bath was set to cool from 0 to $-30{ }^{\circ} \mathrm{C}$ at a rate of $1{ }^{\circ} \mathrm{C} \mathrm{min}-1$, as in a freezing experiment. Figure $\mathrm{S} 4$ shows results of one of the calibration experiments, with the bath temperature $\left(T_{\text {bath }}\right)$ versus the recorded well temperature ( $\left.T_{\text {well }}\right)$ for the nine thermocouples and a line of best fit. The possible pump speeds in the cooling bath are from 1 to 8 , with 8 being the fastest rate of circulation of the coolant. We calculated several parameters to assess the spread of temperatures in the wells: the slope $(m)$ and intercept $(b)$ of the linear fit of the temperature of the bath $\left(T_{\text {bath }}\right)$ versus the averaged temperature of the 9 thermocouples $\left(T_{\text {well,avg }}\right.$ ), the range of temperatures of the 9 thermocouples at $-10{ }^{\circ} \mathrm{C}$ and $-25^{\circ} \mathrm{C}$, and the average of the standard deviations of the thermocouples for $-10{ }^{\circ} \mathrm{C}$ to $-15{ }^{\circ} \mathrm{C}$ and for $-20{ }^{\circ} \mathrm{C}$ to $-25{ }^{\circ} \mathrm{C}$. Table $\mathrm{S} 1$ shows the various combinations of parameters that were tested. We concluded that the best option to minimize the temperature spread across the wells involves using three trays positioned furthest to the right in the bath with the highest pump speed (Table S1, last row entry).

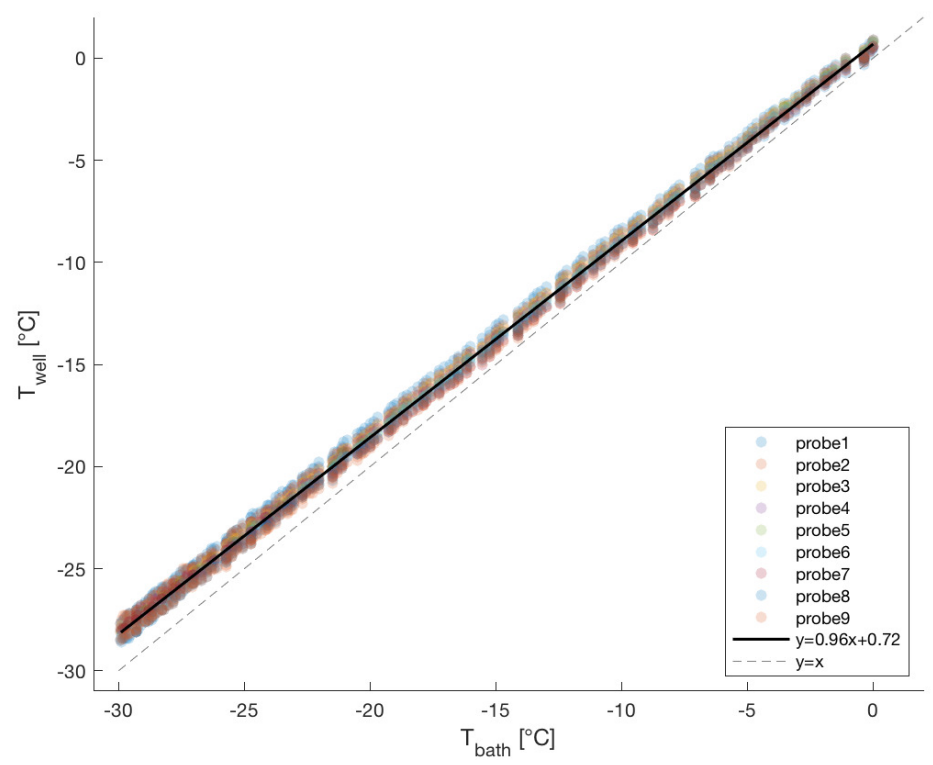

Figure S4. Slope of the Lauda bath temperature calibration experiment. Nine thermocouples were placed in a well filled with ethanol with an even spread across three Piko PCR trays in the cooling bath. Temperature was ramped from 0 to $-30{ }^{\circ} \mathrm{C}$ at a rate of $1{ }^{\circ} \mathrm{C}$ min ${ }^{-1}$. The plot shows the recorded temperatures in the well $\left(T_{\text {well }}\right)$ versus the recorded temperature of the cooling bath $\left(T_{\text {bath }}\right)$. A line of best fit is shown in black, with a slope of 0.96 and a y-intercept of 0.72 . A reference $y=x$ line is shown as a grey dashed line. 
Table S1. Results of testing temperature biases with different number of trays, different tray positions, and varied bath pump speeds. Data presented are of the slope $(m)$ and y-intercept $(b)$ of the calibration equation, the range of temperatures of the thermocouples in all wells at two temperatures, and the average of standard deviations of the thermocouple temperatures in two temperature ranges. Values marked with asterisk (*) are the result of an average of three experiments.

\begin{tabular}{|c|c|c|c|c|c|c|c|c|}
\hline \# of trays & tray position & pump speed & \multicolumn{2}{|c|}{$T_{\text {well,avg }}=m * T_{\text {bath }}+b$} & \multicolumn{2}{|c|}{ range of thermocouples } & \multicolumn{2}{|c|}{ average of standard deviations } \\
\hline \multirow{2}{*}{4} & \multirow{2}{*}{ centered } & 6 & 0.92 & 1.25 & 4.10 & 5.70 & 1.46 & 1.83 \\
\hline & & 8 & 0.94 & 1.01 & 3.00 & 4.90 & 1.11 & 1.56 \\
\hline \multirow{3}{*}{3} & \multirow{3}{*}{$\begin{array}{l}\text { left } \\
\text { middle } \\
\text { right }\end{array}$} & 8 & 0.93 & 0.83 & 2.90 & 6.30 & 1.34 & 2.08 \\
\hline & & 8 & 0.97 & 1.16 & 1.20 & 1.60 & 0.42 & 0.49 \\
\hline & & 8 & $0.95 *$ & $0.75^{*}$ & $1.13 *$ & $1.60 *$ & $0.46^{*}$ & $0.55^{*}$ \\
\hline \multirow{4}{*}{3} & \multirow{4}{*}{ right } & 2 & 0.89 & 2.14 & 2.30 & 3.80 & 0.82 & 1.32 \\
\hline & & 4 & 0.91 & 0.69 & 1.20 & 3.60 & 0.51 & 0.98 \\
\hline & & 6 & $0.92 *$ & $0.79 *$ & $1.40^{*}$ & $2.83^{*}$ & $0.54 *$ & $0.80^{*}$ \\
\hline & & 8 & $0.95 *$ & $0.75^{*}$ & $1.13^{*}$ & $1.60 *$ & $0.46^{*}$ & $0.55^{*}$ \\
\hline
\end{tabular}




\section{S5 Limit of detection}

The limit of detection is presented here, as an example, using 10 background water experiments of 288 -wells each with $5 \mu \mathrm{L}$ volume. A mean frozen fraction curve was calculated by taking the sorted well freezing temperatures and finding the mean at each frozen fraction value from $1-288$. We then calculated a standard deviation at each frozen fraction to obtain a spread of $1 \sigma$ from the mean frozen fraction curve (Fig. S5). Thus, a measured sample has a significant ice nucleating signal if the sample's freezing occurs warmer than the mean $+1 \sigma$. We can also suggest showing a boxplot of the mean frozen fraction (Figure S5).

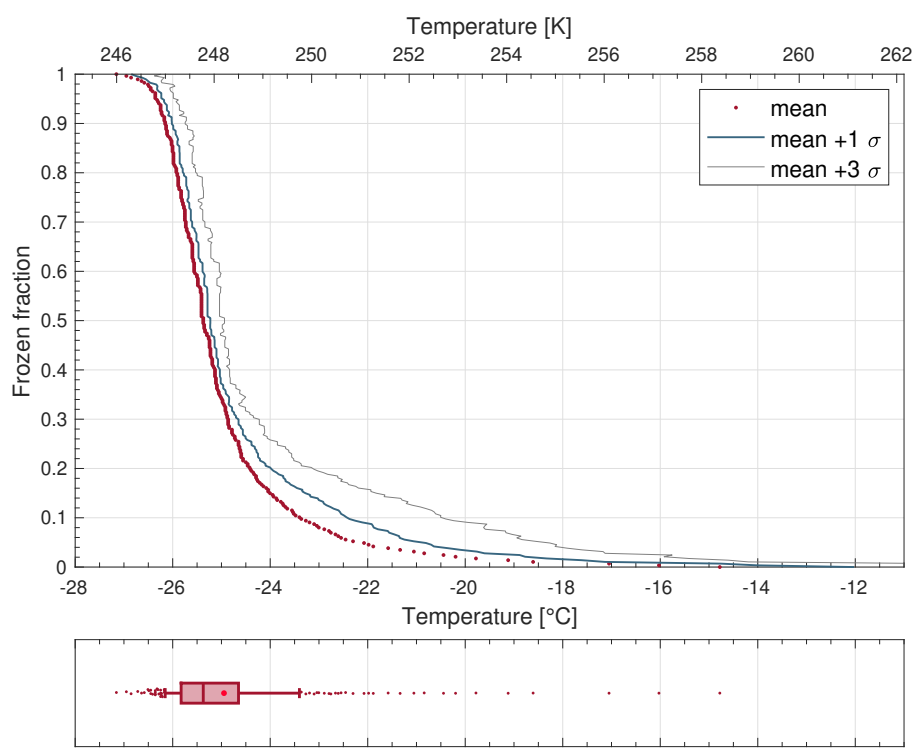

Figure S5. Visual representation of the limit of detection. Top panel shows the mean frozen fraction curve (red dots) with \pm 1 standard deviation (blue lines) and \pm 3 standard deviations (grey lines). These were calculated using 10 experiments of 2885 - $\mu \mathrm{L}$ aliquots of background water. The bottom panel shows the mean frozen fraction represented as a boxplot, where the outer edges of the box are the 25 th and 75 th percentiles, the middle line is the median freezing temperature (i.e. the $T_{50}$ ), the whiskers extend to the 10th and 90th percentiles, the dark red points are the outliers, and the red dot in the box is the mean. All temperature measurements have an uncertainty of $\pm 0.5{ }^{\circ} \mathrm{C}$. 


\section{S6 Volume dependence on non-homogeneous freezing}

55 We measured the water background in FINC with seven different volumes $(5,10,20,30,40,50$ and $60 \mu \mathrm{L}$; Figure S6). Table S2 lists the mean and standard deviations of the temperatures at which 10,50, and $90 \%$ of the droplets froze $\left(T_{10}, T_{50}\right.$, and $T_{90}$, respectively) for each of the volumes. The 5,10 , and $20 \mu \mathrm{L}$ volume mean temperatures are calculated from five experiments, and the $40 \mu \mathrm{L}$ volumes are calculated from three experiments. In general, the $T_{10}, T_{50}$, and $T_{90}$ increases with increasing volume, with the exception of the $20 \mu \mathrm{L}$ volume (Figure S6; Table S2). We investigated possible differences in the surface-area-to-volume ratio (SI Section S9), any differences in surface area does not correlate with the observed freezing. The anomalous and variable freezing behavior of the $20 \mu \mathrm{L}$ volume remains unexplained. We additionally calculated the INP concentration per liter sample volume for every background water experiment (Figure S7). Calculations were done according to Eq. 4 (see main text Section 4.2).
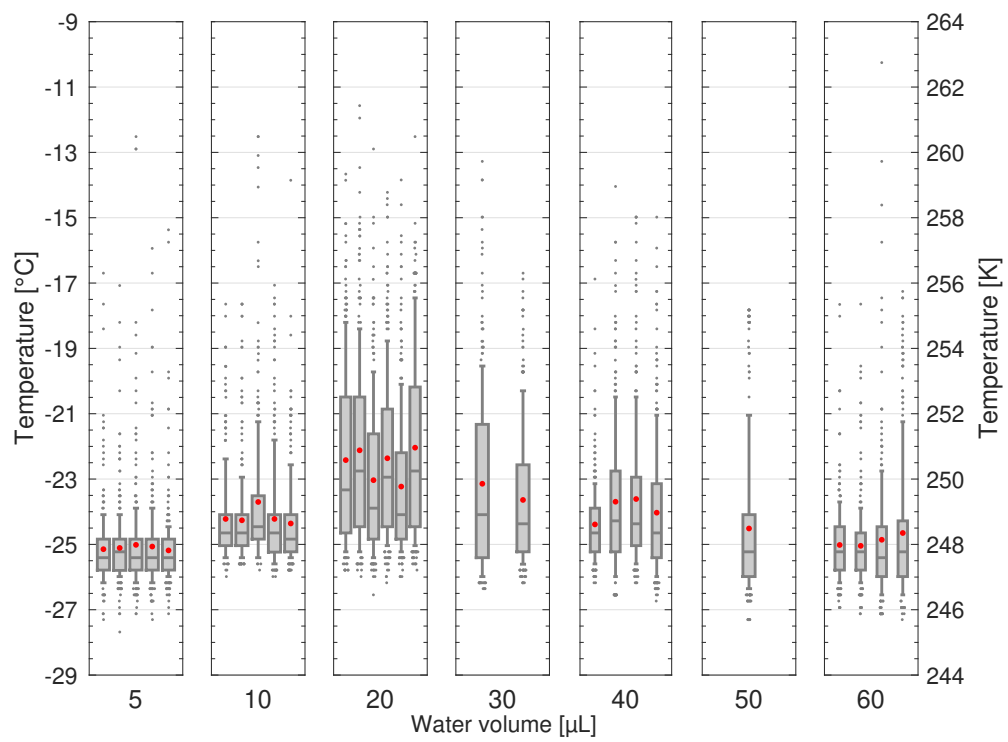

Figure S6. Effect of sample volume on ice nucleating activity of background water in FINC. Each boxplot represents the frozen fraction of 288 wells, where the middle line is the median (i.e. the $T_{50}$ ), the outer edges of the box are the 25 th and 75 th percentiles, the whiskers extend to the 10th and 90th percentiles, and the red dot is the mean. Volumes tested in FINC were 5, 10, 20, 30, 40, 50, and 60 $\mu \mathrm{L}$. All temperature measurements have an uncertainty of $\pm 0.5^{\circ} \mathrm{C}$. 
Table S2. Mean and standard deviations of the $T_{10}, T_{50}$, and $T_{90}$ for each of the volumes of background water in FINC (5, 10, 20, 30, 40, 50, and $60 \mu \mathrm{L}$; for 30 and $50 \mu \mathrm{L}$ there is no standard deviatation because $\mathrm{n}<3$.). $T_{x}$ refers to the temperature at which $\mathrm{x} \%$ of droplets are frozen. All temperature measurements have an uncertainty of $\pm 0.5^{\circ} \mathrm{C}$.

\begin{tabular}{|c|c|c|c|c|c|c|c|c|c|c|c|c|}
\hline \multirow[b]{2}{*}{$\mathrm{T}\left({ }^{\circ} \mathrm{C}\right)$} & \multicolumn{2}{|c|}{$5 \mu \mathrm{L}$} & \multicolumn{2}{|c|}{$10 \mu \mathrm{L}$} & \multicolumn{2}{|c|}{$20 \mu \mathrm{L}$} & \multirow{2}{*}{$\begin{array}{l}30 \mu \mathrm{L} \\
\text { Mean }\end{array}$} & \multicolumn{2}{|c|}{$40 \mu \mathrm{L}$} & \multirow{2}{*}{$\begin{array}{l}50 \mu \mathrm{L} \\
\text { Mean }\end{array}$} & \multicolumn{2}{|c|}{$60 \mu \mathrm{L}$} \\
\hline & Mean & St. Dev. & Mean & St. Dev. & Mean & St. Dev. & & Mean & St. Dev. & & Mean & St. Dev. \\
\hline$T_{10}$ & -23.97 & 0.22 & -22.19 & 0.67 & -18.85 & 0.89 & -19.53 & -21.18 & 1.53 & -19.92 & -22.86 & 1.30 \\
\hline$T_{50}$ & -25.37 & 0.08 & -24.65 & 0.13 & -23.40 & 0.58 & -24.65 & -24.43 & 0.19 & -25.23 & -25.27 & 0.09 \\
\hline$T_{90}$ & -26.10 & 0.10 & -25.45 & 0.08 & -25.23 & 0.13 & -25.45 & -25.73 & 0.22 & -26.36 & -26.36 & 0.21 \\
\hline
\end{tabular}

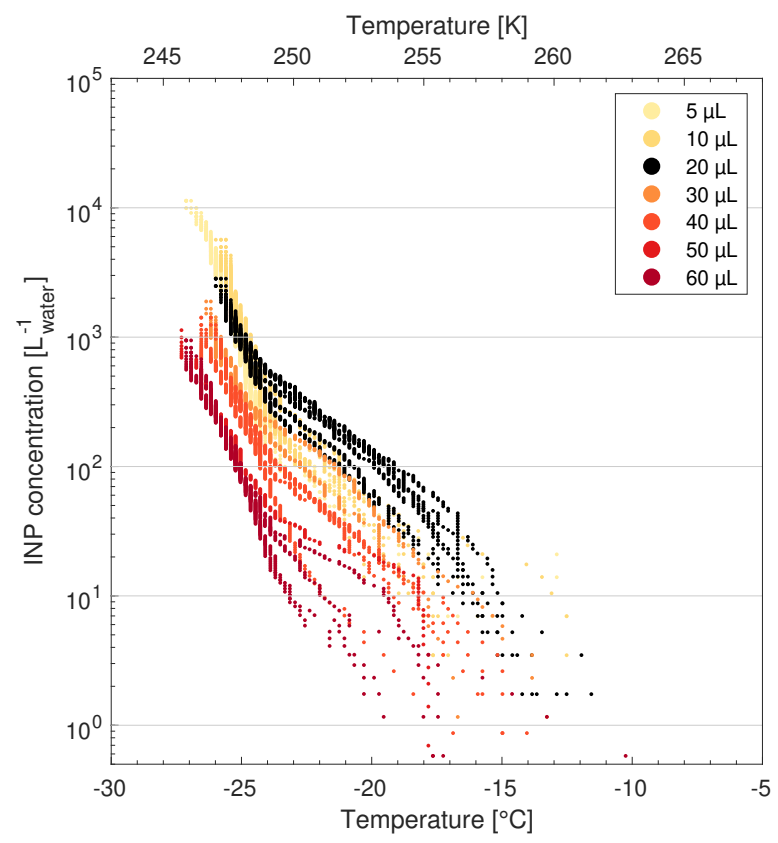

Figure S7. INP concentration per liter of water of background water (molecular biology-free reagent water) measured on FINC. Seven volumes were measured: $5 \mu \mathrm{L}$ to $60 \mu \mathrm{L}$ (markers ranging from light yellow to dark red, respectively), each containing one to six replicates, visually representing Figure S6. The suspicious $20 \mu \mathrm{L}$ experiment is highlighted in black. All temperature measurements have an uncertainty of $\pm 0.5^{\circ} \mathrm{C}$. 


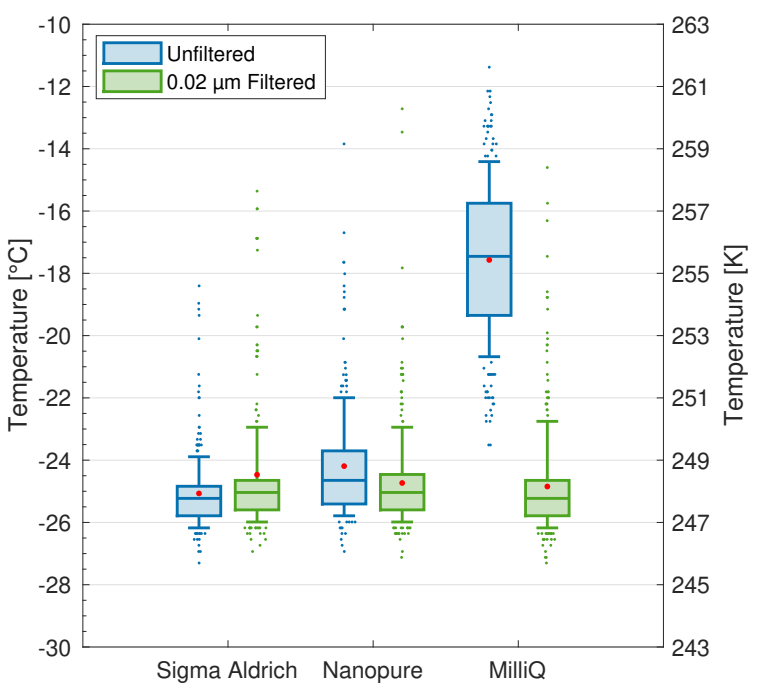

Figure S8. Effect of filtering water through a $0.02 \mu \mathrm{m}$ filter on ice nucleating activity. Filtered water samples include the microbiology-free reagent water from Sigma Aldrich (left), our lab's water from the Nanopure system (middle), and our lab's water from the. Milli-Q system (right). Blue boxplots represent the freezing of unfiltered water samples, and green boxplots represent the freezing of water samples filtered through a $0.02 \mu \mathrm{m}$ filter. 
Since we do not use a tray cover film during the measurement, water vapor may possibility condense into the tray as the temperature cools. Therefore, we estimated the volume of condensable water vapor between the plexiglass cover and the Piko PCR trays. The volume of air between the plexiglass cover and the trays is $2.704 * 10^{-4} \mathrm{~m}^{3}$ (Eq. S1). We consider here a case representative of an upper limit volume of water. At $0^{\circ} \mathrm{C}$ and at $1013 \mathrm{hPa}(1 \mathrm{~atm})$, the water vapor pressure at $90 \% \mathrm{RH}$ is 611.2 $70 \mathrm{~Pa}$ (Eq. S2, which equated to $1.76 * 10^{-7}$ moles of water (Eq. S3). These moles of water total a liquid volume of $2 \mathrm{~nL}$ (Eq. S4. This volume of water in the slab of air above 288 wells is negligible in comparison to the 5-60 $\mu \mathrm{L}$ sample volumes per well. We therefore conclude that condensed water from the above air will not affect the freezing measurement.

$V_{\text {air }}=0.130 \mathrm{~m} * 0.130 \mathrm{~m} * 0.016 \mathrm{~m}=2.704 * 10^{-4} \mathrm{~m}^{3}$

$P_{\text {water }}=R H * P_{\text {sat }, \mathrm{H} 2 \mathrm{O}}$

$N_{\text {moles water }}=P_{\text {water }} * V_{\text {air }} /(R * T)$

$V_{\text {condensed water }}=N_{\text {moles water }} * 12 \mathrm{~g} / \mathrm{mol} * 1 \mathrm{~mL} / \mathrm{g}$

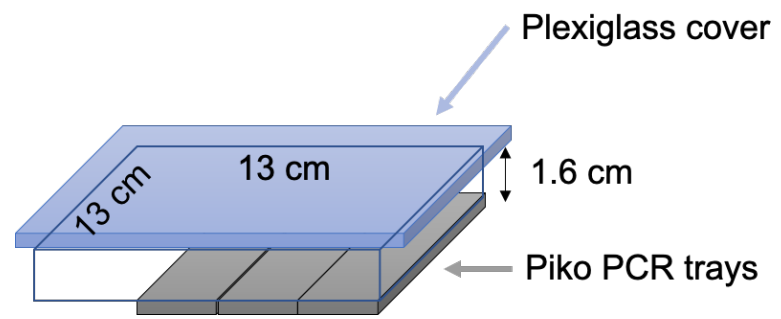

Figure S9. Figure of the slab of air 


\section{S9 Well surface area calculations}

The anomalous freezing behavior of $20 \mu \mathrm{L}$ volumes of water, compared to the other volumes (Fig. S6; Table S2), led us to consider a possible effect of the surface-area-to-volume ratio on ice nucleation. The dimensions of the wells of the Piko PCR tray were in part taken from the technical drawing provided by ThermoFisher (2014), and the missing dimensions (in blue in Fig. S10) were found using image analysis with ImageJ software (Abràmoff et al., 2004). The well shape was estimated as two truncated cones (Fig. S10). Therefore, depending on the volume of the solution in a well different equations were required to calculate the surface area of the solution in contact with the well. It is important to note that the presence of the meniscus was omitted for the following calculations. Firstly, through the use of similar triangles, the radius of the solution in the well $\left(r_{\text {sol }}\right)$ for a given height $d h$ in the lower truncated cone could be calculated using:

$r_{\text {sol }}=d h *\left(r_{\text {up }}-r_{\text {base }}\right) / h_{\text {cone }}+r_{\text {base }}$

where $r_{\text {up }}, r_{\text {base }}$ and $h_{\text {cone }}$ are the radius of the top and bottom, and the height of the lower truncated cone, respectively. $r_{\text {sol }}$ was calculated for varying $d h$ until the volume of solution in the lower truncated cone $\left(V_{\text {calc }}\right)$, calculated using Eq. S6, matched the volume of solution pipetted into the well.

$V_{\text {calc }}=(\pi / 3) *\left(r_{\text {sol }}^{2}+r_{\text {sol }} * r_{\text {base }}+r_{\text {base }}^{2}\right) * d h$

For the solutions of $20 \mu \mathrm{L}$ and larger, the upper truncated cone needed to be considered. Therefore, the volume of the lower truncated cone (calculated using Eq. S6 but with the values from Fig. S10) was subtracted from the pipetted volume and $r_{\text {sol }}$ was calculated similarly to as in Eq. S5 except with the dimensions of the upper truncated cone as follows:

$r_{\mathrm{sol}}=d h *\left(r_{\mathrm{top}}-r_{\mathrm{up}}\right) / h_{\mathrm{Ucone}}+r_{\mathrm{up}}$

where $r_{\text {top }}$ and $h_{\text {Ucone }}$ are the radius of the top and the height of the upper truncated cone. Note $r_{\text {up }}$ is the same in Eqs. S5 and $\mathrm{S} 7$ as the radius of the top of the lower truncated cone is the same as the radius of the bottom of the upper truncated cone. $r_{\text {sol }}$ for increasing heights was calculated until the volume of solution in the upper truncated cone (Eq. S6 with $r_{\text {base }}$ replaced by $r_{\text {up }}$ ) plus the total volume of the lower truncated cone was equal to the pippeted solution in the well. With $r_{\text {sol }}$ and $d h$ found for each volume of pipetted solution, the surface area of solution in contact with the well surface $\left(S A_{\text {well }}\right)$ was calculated as follows for 5 and $10 \mu \mathrm{L}$ volumes:

$S A_{\text {well }}=\pi *\left(r_{\text {sol }}+r_{\text {base }}\right) * \sqrt{\left(r_{\text {sol }}-r_{\text {base }}\right)^{2}+d h^{2}}+\pi * r_{\text {base }}^{2}$

and $20-60 \mu \mathrm{L}$ volumes: 
$S A_{\text {well }}=\pi *\left(r_{\text {sol }}+r_{\text {up }}\right) * \sqrt{\left(r_{\text {sol }}-r_{\text {up }}\right)^{2}+d h^{2}}+\pi *\left(r_{\text {up }}+r_{\text {base }}\right) * \sqrt{\left(r_{\text {up }}-r_{\text {base }}\right)^{2}+h_{\text {cone }}^{2}}+\pi * r_{\text {base }}^{2}$

The surface area of solution exposed to air $\left(S A_{\text {air }}\right)$ for each pipetted volume was calculated using:

$S A_{\text {air }}=\pi * r_{\text {sol }}^{2}$

The calculated values for $r_{\mathrm{sol}}, d h, S A_{\mathrm{well}}, S A_{\mathrm{air}}$, as well as the ratios of surface area exposed to the well and air as a function of volume are shown in Table S3. The values in Table S3 and the volume versus surface area lines in Fig. S11 show that there is no clear explanation for the anomalous behaviour of the $20 \mu \mathrm{L}$ solution.

Table S3. Properties of the 5, 10, 20, 30, 40, 50, and $60 \mu \mathrm{L}$ solutions in the piko well tray. The radius $r_{\text {sol }}$ and height $d h$ of the solution in the well is shown. Additionally, the surface area of the solution in contact with the well $S A_{\text {well }}$ and air $S A_{\text {air }}$, as well as the surface to volume $S A_{\text {well }} / V$ and air to volume $S A_{\text {air }} / V$ ratios are displayed.

\begin{tabular}{lllllll}
\hline Volume $\left(\mathrm{mm}^{3}\right)$ & $r_{\text {sol }}(\mathrm{mm})$ & $d h(\mathrm{~mm})$ & $S A_{\text {well }}\left(\mathrm{mm}^{2}\right)$ & $S A_{\text {air }}\left(\mathrm{mm}^{2}\right)$ & $S A_{\text {well }} / V\left(\mathrm{~mm}^{-1}\right)$ & $S A_{\text {air }} / V\left(\mathrm{~mm}^{-1}\right)$ \\
\hline 5 & 1.05 & 2.16 & 13.07 & 3.47 & 2.61 & 0.69 \\
10 & 1.27 & 3.34 & 21.80 & 5.07 & 2.18 & 0.51 \\
20 & 1.47 & 4.95 & 36.19 & 6.79 & 1.81 & 0.34 \\
30 & 1.52 & 6.38 & 49.60 & 7.21 & 1.65 & 0.24 \\
40 & 1.56 & 7.73 & 62.62 & 7.62 & 1.57 & 0.19 \\
50 & 1.60 & 9.01 & 75.31 & 8.01 & 1.51 & 0.16 \\
60 & 1.64 & 10.23 & 87.69 & 8.40 & 1.46 & 0.14 \\
\hline
\end{tabular}



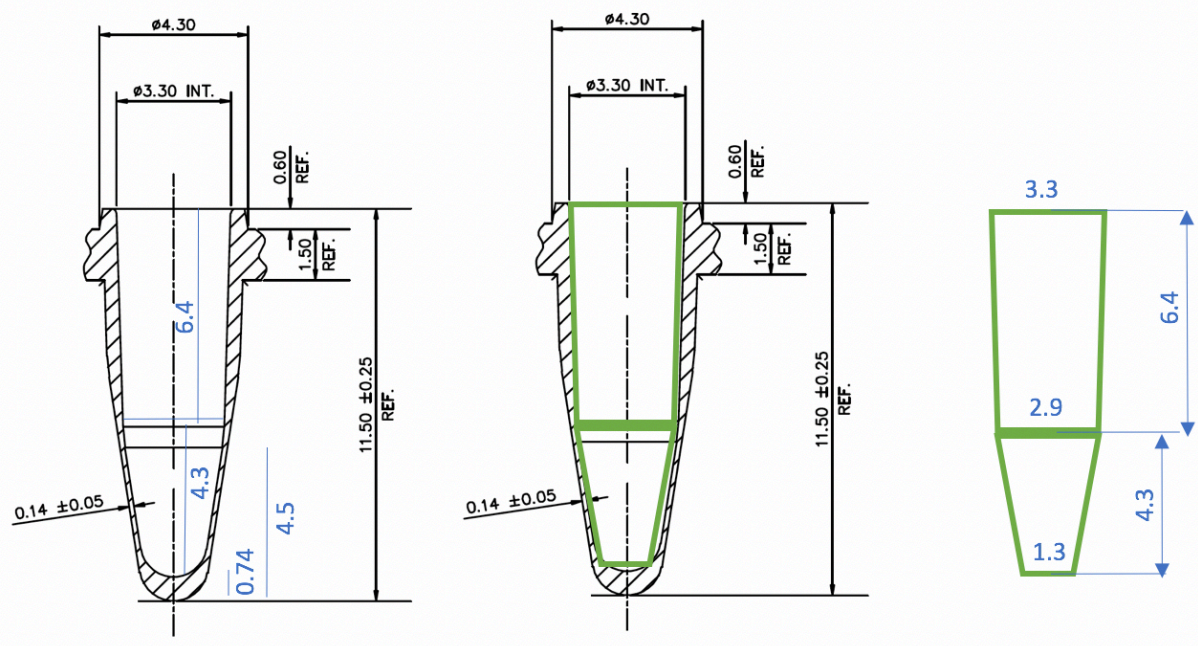

Figure S10. Diagrams showing shape and dimensions of a well in the Piko PCR trays used in FINC. The base diagrams (all black lines and numbers) are from the technical drawing provided by ThermoFisher (2014) and colored lines and numbers are added from image analysis in Image J to show dimensions needed for calculated surface area. 

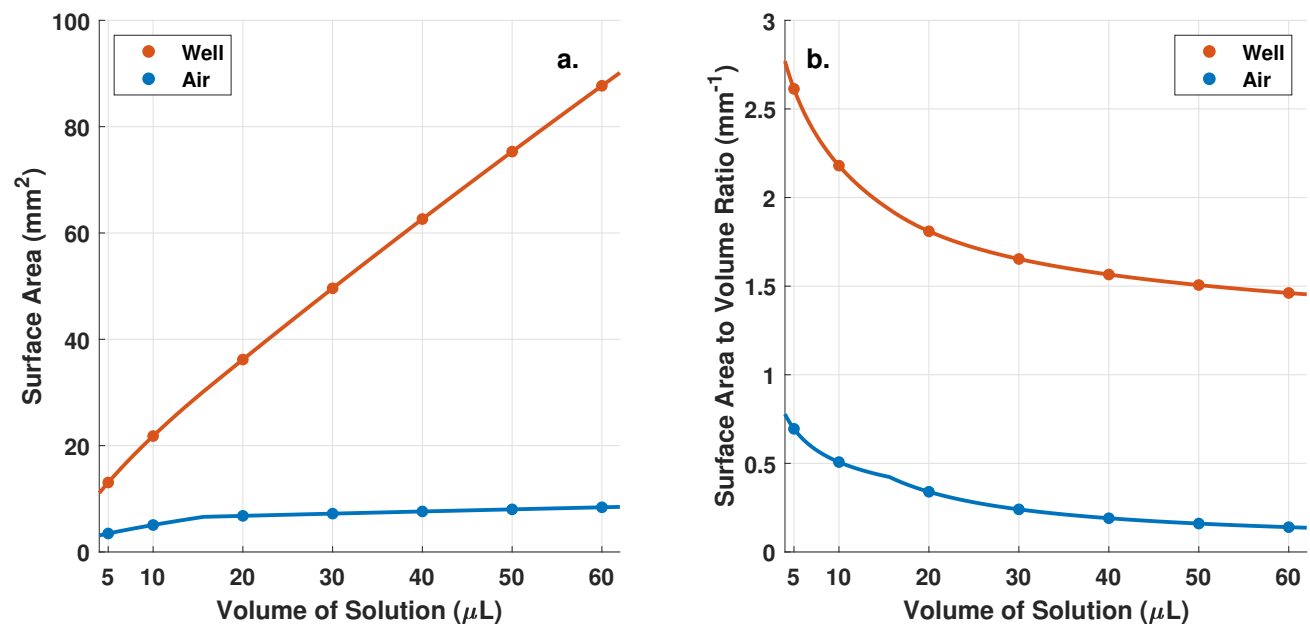

Figure S11. Surface area to the well surface (red) and air (blue) for each solution volume tested (a). The ratio of solution volume to surface area exposed to the well surface (red) and air (blue) for each solution volume tested (b) 


\section{S10 Effect of bubbles in the well}

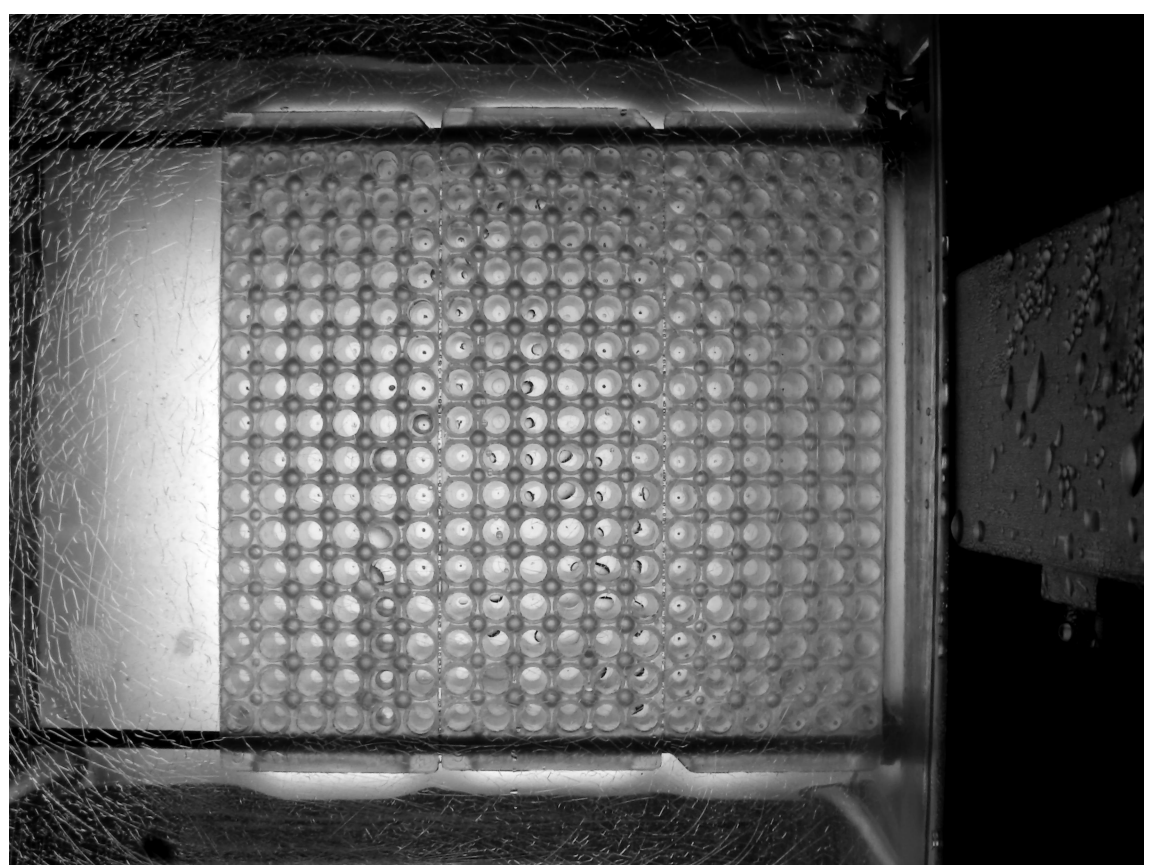

Figure S12. Example of air bubbles present in the Piko PCR trays. These bubbles lead to warmer freezing and must be avoided during experiments. The corresponding frozen fraction curves of each tray are in Figure S13 


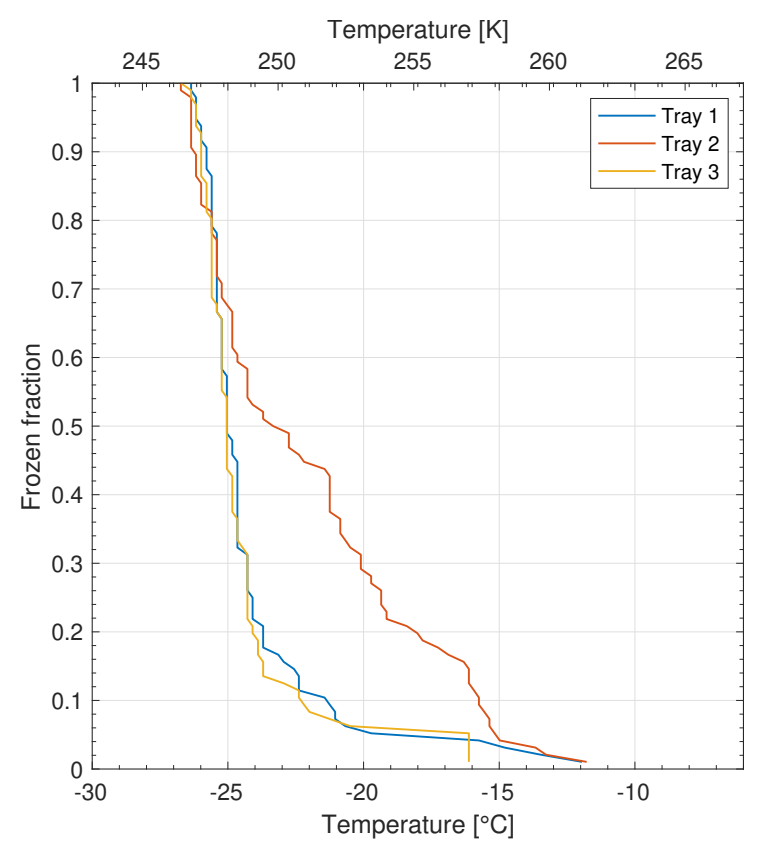

Figure S13. Frozen fraction curves for the three trays shown in Figure S12. Tray 1 (blue) is the leftmost tray, Tray 2 (red) is the middle tray, and Tray 3 (yellow) is the rightmost tray.

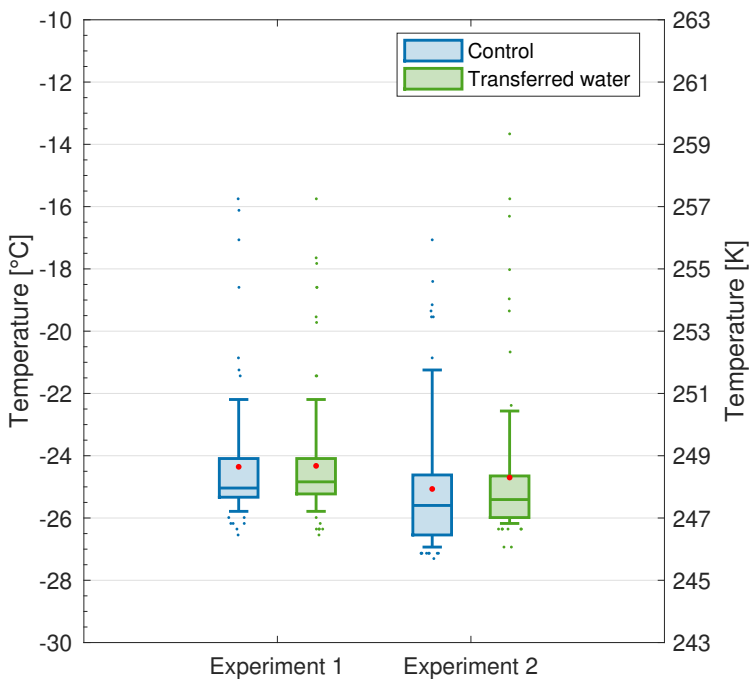

Figure S14. Experiments showing lack of contamination introduced from the wells. 


\section{S11 Lignin batch specifications}

The lignin used in the experiments presented in this study was alkali low-sulfonate kraft lignin (471003, Sigma Aldrich). Three different batches of this product were bought, and their differences in ice nucleating activity were tesed. Table S4 lists the

115 details of the three batches bought, including batch number, production date, carbon content, and sulfur content, provided by their respective Certificate of Analyses (Sigma-Aldrich).

Table S4. Specifications of the three batches of lignin used, including batch number, dates of production, and carbon and sulfur content. All batches were bought at Sigma Aldrich, product number 471003, and these specifications are provided in their Certificate of Analyses.

\begin{tabular}{lllll}
\hline Batch & Number & Produced & Carbon \% & Sulfur \% \\
\hline 1 & 04414PE & Nov 2006 & 50.13 & 3.33 \\
2 & MKCL2371 & Nov 2019 & 47.8 & 1.7 \\
3 & MKCK3344 & July 2019 & 50.8 & 2.4 \\
\hline
\end{tabular}




\section{S12 Lignin solution stability and storage}

A concentrated lignin solution at $200 \mathrm{mg} \mathrm{C} \mathrm{L}^{-1}$ was measured over two weeks to test how the ice nucleating activity changed over time. After solution preparation, the solution was stored in the fridge. We found no significant difference in freezing temperatures for the solution measured in FINC over two weeks (Figure S15).

Futhermore, the impact of the storage method of lignin solutions on its ice nucleating activity was tested by comparing solutions stored in the fridge versus at room temperature (Figure S16). Solutions of lignin Batch 2 and Batch 3 (see Section S11) were prepared at $20 \mathrm{mg} \mathrm{C} \mathrm{L}^{-1}$ and each split into two containers, one to be stored in the fridge and one on the lab bench (both stored covered with aluminum foil, protected from light). A bottle of background water was also prepared identically as a control. The ice nucleating activity of each solution was measured on FINC on the day of preparation and again 24 hours later, after storage in their respective locations. We found no significant difference between storage conditions, and no significant difference between the ice nucleating activity on the day of or the day after solution preparation.

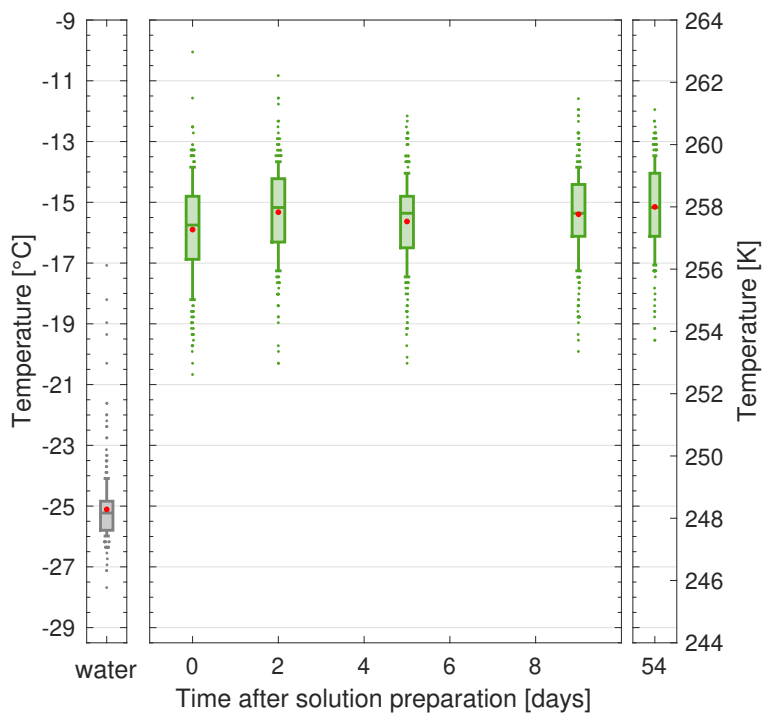

Figure S15. Solution stability over 10 days of a $200 \mathrm{mg} \mathrm{C} \mathrm{L}^{-1}$ lignin solution (green boxplots). Lignin used was of Batch 2 (corresponds to batch number MKCL2371, 471003, Sigma Aldrich). Shown for comparison is the background water frozen fraction box plot (grey boxplot). Each boxplot represents the frozen fraction of the 288 wells of each freezing experiment, where the middle line is the median (i.e. the $T_{50}$ ), the outer edges of the box are the 25 th and 75 th percentiles, the whiskers extend to the 10th and 90th percentiles, and the red dot is the mean. All temperature measurements have an uncertainty of $\pm 0.5^{\circ} \mathrm{C}$. 


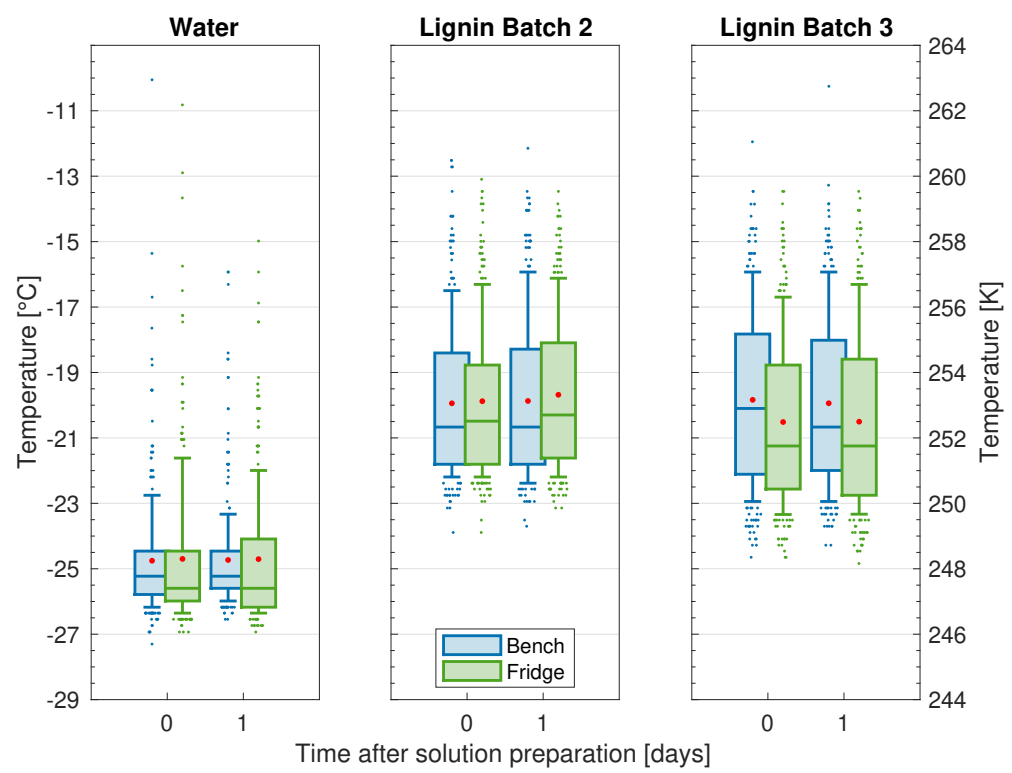

Figure S16. Lignin solutions and background water freezing temepratures on the day of preparation and one day after preparation, stored either in the fridge (green boxplots) or on the lab bench (blue boxplots). Lignin used was of Batch 2 and Batch 3 (corresponds to batch numbers MKCL2371 and MKCK3344 of product number 471003 from Sigma Aldrich). Each boxplot represents the frozen fraction of the 288 wells of each freezing experiment, where the middle line is the median (i.e. the $T_{50}$ ), the outer edges of the box are the 25 th and 75th percentiles, the whiskers extend to the 10th and 90th percentiles, and the red dot is the mean. All temperature measurements have an uncertainty of $\pm 0.5^{\circ} \mathrm{C}$.

\section{References}

Abràmoff, D. M. D., Magalhães, P. J., and Ram, S. J.: Image Processing with ImageJ, Biophotonics International, 11, 36-42, 2004.

David, R. O., Cascajo-Castresana, M., Brennan, K. P., Rösch, M., Els, N., Werz, J., Weichlinger, V., Boynton, L. S., Bogler, S., BorduasDedekind, N., Marcolli, C., and Kanji, Z. A.: Development of the DRoplet Ice Nuclei Counter Zurich (DRINCZ): Validation and Application to Field-Collected Snow Samples, Atmos. Meas. Tech., 12, 6865-6888, https://doi.org/10.5194/amt-12-6865-2019, 2019.

Sigma-Aldrich: Lignin, Alkali 471003, https://www.sigmaaldrich.com/catalog/product/aldrich/471003.

ThermoFisher: Technical Drawing: Piko PCR Plate, 96-Well, Clear., https://www.thermofisher.com/order/catalog/product/SPL096050\#/SPL096050, 